\title{
Invasive benign meningioma: Clinical characteristics, surgical strategies and outcomes from a single neurosurgical institute
}

\author{
QINGTANG LIN, FENG LING and GENG XU \\ Department of Neurosurgery, Xuanwu Hospital, Capital Medical University, Beijing 100054, P.R. China
}

Received January 4, 2015; Accepted February 18, 2016

DOI: 10.3892/etm.2016.3223

\begin{abstract}
The aim of the present study was to improve the prognosis for patients with invasive benign meningioma by increasing the precision of pre-operative evaluation and refining the surgical resection strategy. A retrospective review of all the cases of invasive benign meningioma admitted to a single institute from 2005 to 2010 was conducted. The clinical characteristics, magnetic resonance imaging (MRI) findings, refined surgical strategies and outcomes were summarized and analyzed. There were 19 cases of invasive benign meningioma among 254 cases of meningioma. Of the earliest 4 cases, a traditional extra-capsular surgical removal approach was applied, which resulted in permanent neurological deficits for all cases. A modified surgical strategy combining intra- and extra-capsular resection techniques was employed for the subsequent 15 cases, which lead to mild neurological impairment in only 1 case. Pre-operative recognition of this unique sub-type of meningioma maybe facilitated by its typical MRI study findings, and the combination of intra- and extra-capsular resection techniques may be of critical importance in achieving complete tumor removal while maintaining intact neurological functions.
\end{abstract}

\section{Introduction}

Meningioma is the second most common primary brain tumor, which accounts for $13-15 \%$ of all intracranial neoplasms $(1,2)$. Traditionally, it is classified by the combination of two properties: The site of the tumor (for example, convex meningioma, sphenoid crest meningioma or cerebellar meningioma) and the pathological nature of the tumor (benign or malignant meningioma). This classification provides accurate localization of the tumor and enables the selection of the appropriate

Correspondence to: Dr Qingtang Lin or Professor Geng Xu, Department of Neurosurgery, Xuanwu Hospital, Capital Medical University, 45 Changchun Street, Xicheng, Beijing 100054, P.R. China

E-mail: kingsang2002@hotmail.com

E-mail: xugeng2006@sina.com

Key words: meningioma, benign, invasive, intra-capsular, extra-capsular surgical approach, in addition to allowing the multidisciplinary management plan and the patient's prognosis to be determined $(3,4)$. In general, the pathological nature of meningioma determines its association with the brain parenchyma, which is that benign meningioma is usually compressive to the brain parenchyma due to its expansive growth, and malignant meningioma is invasive into the neighboring brain parenchyma due to its intrusive growth $(5,6)$. However, clinical observations have indicated that there is a sub-group of benign meningioma displaying a malignant growth pattern, that is, invasion into the neighboring brain tissue (7-11). This sub-type of meningioma is usually unnoticed prior to surgical resection by the neurosurgeon, which can often result in damage of the neighboring brain tissue. To improve our knowledge about this unique type of meningioma and facilitate the preservation of the neighboring brain parenchyma, a retrospective review of all the cases of invasive benign meningioma in a single hospital during the past 6 years was conducted. This review presents their clinical characteristics, the evolving treatment strategies and the post-operative outcomes.

\section{Materials and methods}

From August 2005 to August 2010, there were 254 patients with meningioma who were treated surgically in Xuanwu Hospital affiliated with Capital Medical University (Beijing, China). Among these, there were 19 cases (7.4\%) of invasive benign meningioma. The clinical characteristics, magnetic resonance imaging (MRI) findings, refined surgical strategies and outcomes were summarized and analyzed. This retrospective study was approved by the Ethics Committee of Xuanwu Hospital affiliated with Capital Medical University. Written informed consent was obtained from each patient.

The clinical characteristics, magnetic resonance imaging (MRI) results, refined surgical strategies and outcomes were summarized and analyzed. MRI data were acquired using a 1.5 T Siemens Sonata scanner (Siemens Medical Solutions USA, Inc., Malvern, PA, USA). Standard neurological physical examination was conducted to evaluate the overall function of the central nerve system of the patients.

\section{Results}

Clinical and MRI characteristics. The mean age of the 19 patients with invasive benign meningioma was 53 years 


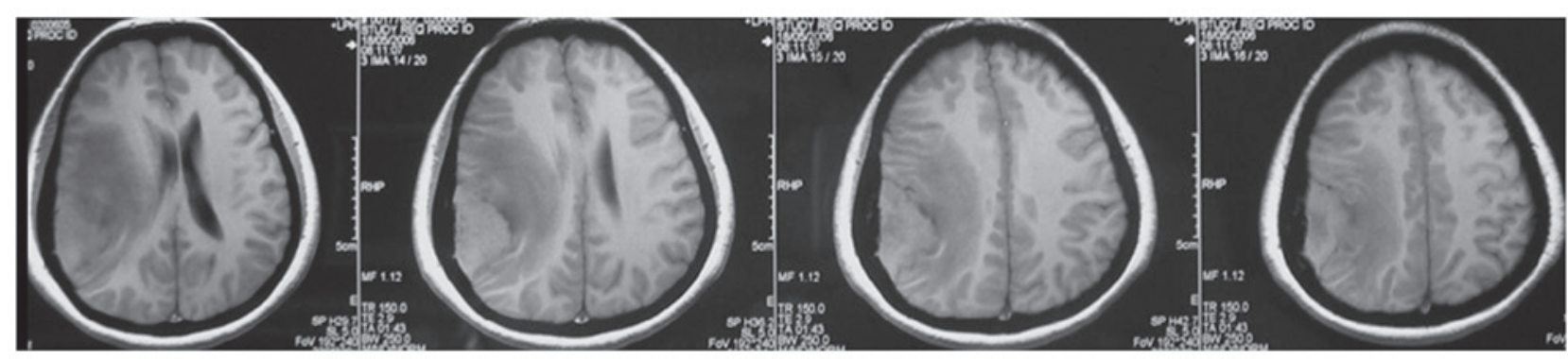

Figure 1. T1-weighted magnetic resonance images demonstrated that there was an extrinsic space-occupying lesion located in the right fronto-tempo-parietal lobes. The lesion was iso to hypo in signal intensity and grew along the dural matter.

(range, 40-72 years). This cohort consisted of 9 male patients and 10 female patients. The initial symptoms at diagnosis included decreased muscle power of a single extremity (7 cases), dysphasia accompanied by decreased muscle power of a single extremity (9 cases) and decreased sensation of a single extremity (3 cases). Notably, to some extent, there were certain common features shared by the MRI results of these patients. The meningiomas were all located on the convexity of the cerebral hemisphere and crossed the central lobe. On T1-weighted imaging (Fig. 1), the meningiomas appeared to exhibit iso- or hyperintense signals and grew along the dural matter. On T2-weighted imaging (Fig. 2), there was absent or very narrow sub-arachnoid space between the meningioma and the adjacent brain parenchyma. Fluid-attenuated inversion recovery imaging (Fig. 3) confirmed the existence of severe edema surrounding the tumor. On T1-weighted enhanced images (Figs. 4 and 5), firstly, these meningiomas were flat in shape with extensive bases, accompanied by evident 'tail signs'; secondly, at the apex of the meningiomas, the boundary between the tumors and the adjacent brain parenchyma could not be perceived clearly; thirdly, these meningiomas appeared to be protruding into the neighboring brain tissue.

Surgical strategies and outcomes. Microsurgeries were performed to remove the meningioma for all 19 cases. For all cases, the originating site of the meningioma was coagulated and divided first. Following this, for the earliest 4 cases, a traditional extra-capsular approach was used, which involved separating the tumor from the brain parenchyma along the arachnoid membrane. In two of these 4 cases, it was observed that there were 'cauliflower-like' nodules on the surfaces of the meningiomas, which enwrapped the normal brain tissues and vessels. This surgical challenge was sometimes not possible to overcome and the total removal of the meningioma could only be achieved at the cost of the enwrapped normal brain tissue and associated vessels. To improve the surgical removal, the strategy was modified for the following 15 cases. Initially, the intra-capsular extirpation of the central part of the meningioma was conducted carefully, until the capsule wall of the tumor was reached. Then, dissection of the tumor was continued according to the classic extra-capsular method, which was along the interface between the tumor and the brain parenchyma. In 11 of these 15 cases, the 'cauliflower-like' tumor nodules enwrapping the neighboring normal cortex and associated vessels were also encountered. These enwrapped brain structures could be much more easily separated and spared by gentle dissection, which was greatly facilitated by the removal of the bulk of the tumor. For all 19 cases, total removals were achieved and the pathologic results were benign meningioma [World Health Organization (WHO) grade 1-2]. The follow-up period was $>6$ months. For the earliest 4 cases, mild but permanent neurological impairments were observed, which included mild dysphasia (1 case), decreased muscle power of the contralateral fingers ( 2 cases) and decreased sensation of the contralateral palms (1 case). By contrast, for the other 15 cases, there was only 1 case with permanent neurological impairment (decreased sensation and muscle power of the contralateral fingers).

\section{Discussion}

The traditional pathological classification of meningioma consists of benign (WHO 1), atypical (WHO 2) and anaplastic types (WHO 3) (12). In general, benign meningioma grows expansively and compresses the neighboring brain parenchyma while aggressive meningioma is invasive. However, clinical practice has revealed that there is a sub-group of meningioma with intermediate characteristics. These meningiomas are benign in histology, but malignant in their growth (13). This introduces the challenge of total removal while ensuring the protection of neurological functions. Previous studies have demonstrated the MRI features and potential molecular biomarker for this sub-group of meningiomas $(13,14)$. However, there are no prior studies describing the overall clinical features for these patients and surgical strategy for the removal of these tumors.

According to the experience gained in these 19 cases, it was observed that there were certain features shared by these invasive benign meningiomas. The peak age of onset was $\sim 50$ years; the main manifestation was mild focal neurological deficits, which included dysphasia, and decreased sensation and muscle power of the contralateral limb. The MRI findings usually had the following characteristics: The lesions were located at the convexity of the cerebral hemisphere involving the central lobe, with an extensive base at the dural matter and evident 'tail sign'; there was a minimal boundary between the tumor and the neighboring brain cortex; finally, and probably most importantly, the apex of tumor often enwrapped the normal brain tissue and associated vessels. Due to the malignant growth, it was challenging to completely remove this benign meningioma while ensuring that neurological function remained intact. Resection of the earliest 4 cases was 


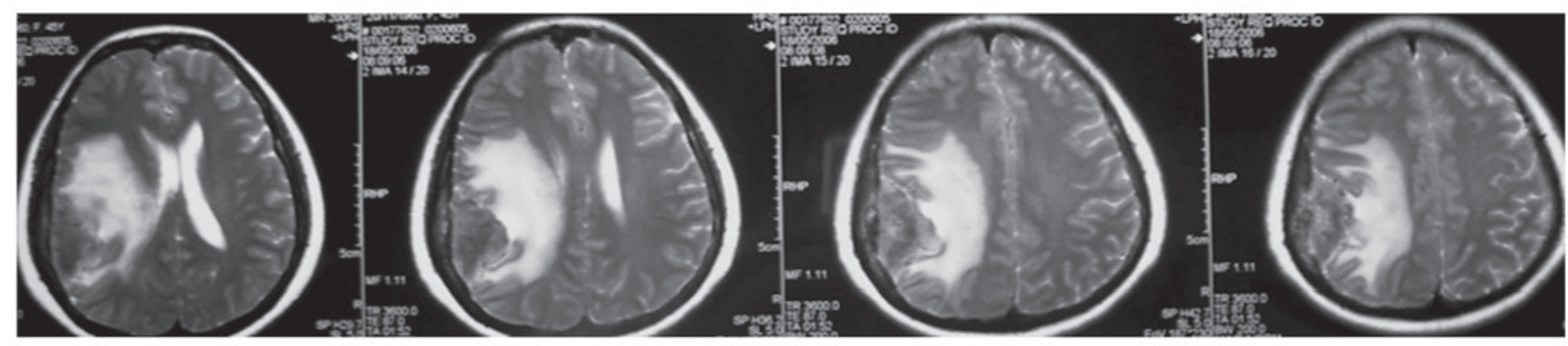

Figure 2. T2-weighed magnetic resonance images demonstrated that there was absent or very narrow space between the lesion and the neighboring brain parenchyma. The lesion was iso to hypo in signal intensity and surrounded by severe edema.

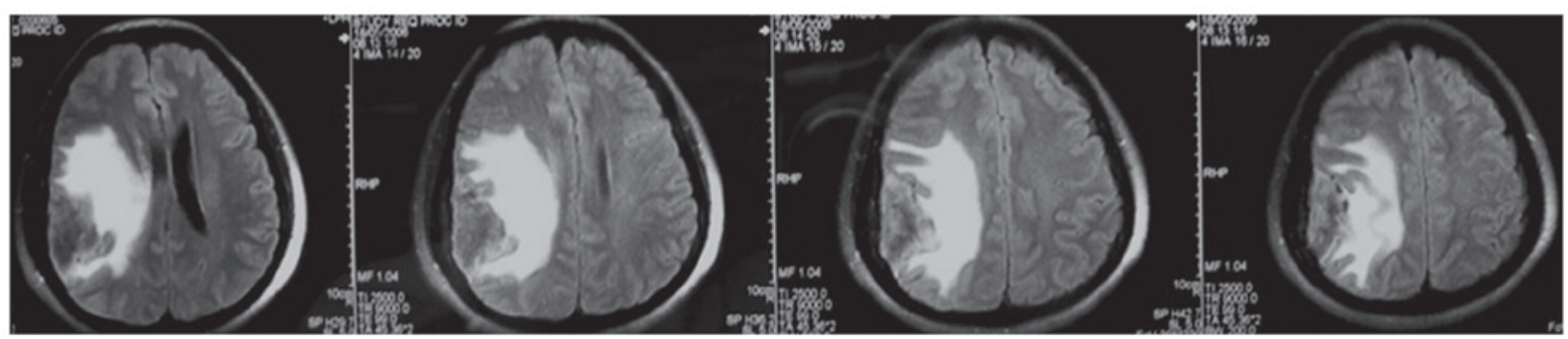

Figure 3. Fluid-attenuated inversion recovery images confirmed the existence of edema surrounding the lesion.

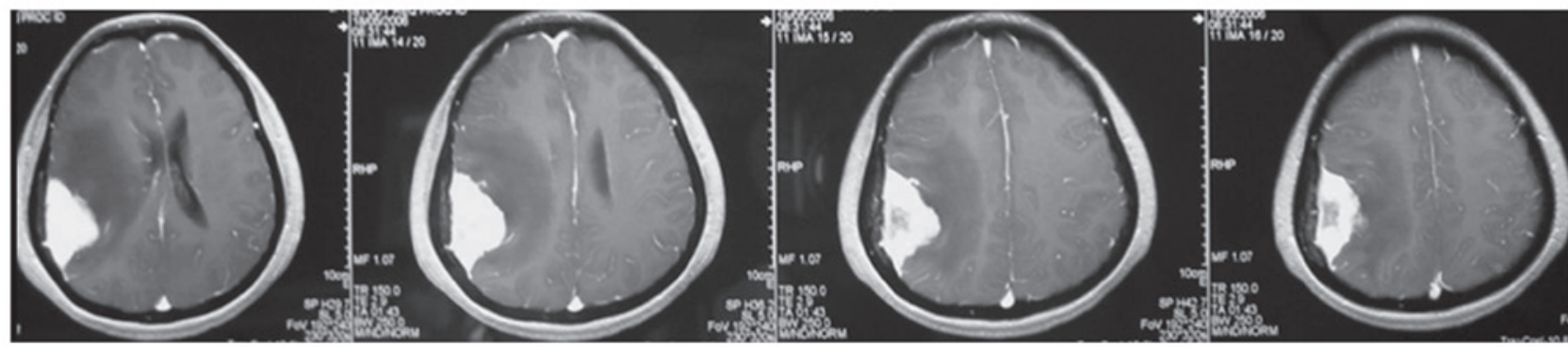

Figure 4. Axial view of the T1 contrast imaging revealed that the lesion was enhanced homogeneous and significantly, with an extensive base and a 'tail sign' at each side. At the apex of the lesion, there was no perceivable boundary between the lesion and the adjacent brain parenchyma and it appeared that there were tumor nodules enwrapping the neighboring brain tissues.

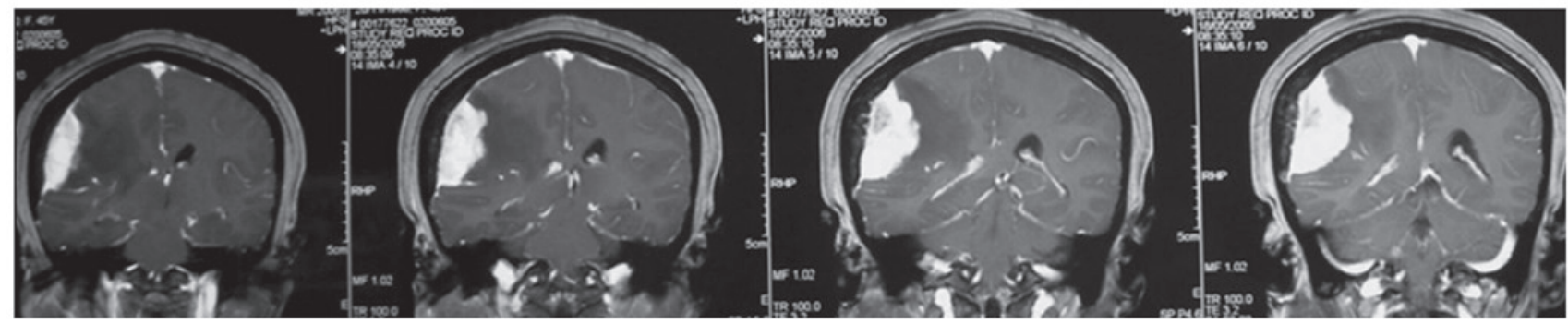

Figure 5. Coronal view of the T1 contrast imaging showed the flat shape of the lesion and the 'cauliflower like' tumor nodules at the tumor apex.

performed according to the traditional extra-capsular strategy, which was to coagulate and divide the tumor base first, and then continue the dissection along the interface between the tumor and brain parenchyma. This approach inevitably damaged the vessels transiting from and into the tumor. The observation that all 4 cases had permanent neurological deficits confirmed the disadvantage associated with this surgical strategy. Following careful analysis of the surgical outcomes, the resection method was modified by combining intra- and extra-capsular approaches. The first step was the same as the classical method, which was to coagulate and divide the tumor base. Afterwards, intra-capsular extirpation of the central part of the tumor was performed. Care was taken not to damage the transit vessels when approaching the tumor-brain interface. The enucleation of the central part of the tumor created a working space, which greatly facilitated the identification of the transit vessels. After 
this, the tumor was separated from brain parenchyma along the sub-arachnoid membrane. The use of a sponge during this dissection process was important. Finally, it was critical to separate the enwrapped brain cortex and associated vessels from the invading 'cauliflower-like' nodules of the meningioma. It is recommended that no efforts are spared in this process, since the enwrapped brain tissue may have retained its ability to function. The observation that there was only 1 case with mild neurological impairment post-operatively in the later 15 cases confirms the advantage of the modified strategy.

In summary, the present study further revealed the clinical features of the invasive benign meningioma and indicated the advantage of combined intra-extra capsular strategy for the surgical resection.

\section{References}

1. Wiemels J, Wrensch M and Claus EB: Epidemiology and etiology of meningioma. J Neurooncol 99: 307-314, 2010.

2. Longstreth WT Jr, Dennis LK, McGuire VM, Drangsholt MT and Koepsell TD: Epidemiology of intracranial meningioma. Cancer 72: 639-648, 1993.

3. Mawrin C and Perry A: Pathological classification and molecular genetics of meningiomas. J Neurooncol 99: 379-391, 2010.

4. Scheithauer BW: Tumors of the meninges: Proposed modifications of the World Health Organization classification. Acta Neuropathol 80: 343-354, 1990.
5. Riemenschneider MJ, Perry A and Reifenberger G: Histological classification and molecular genetics of meningiomas. Lancet Neurol 5: 1045-1054, 2006.

6. Modha A and Gutin PH: Diagnosis and treatment of atypical and anaplastic meningiomas: A review. Neurosurgery 57: 538-550, 2005.

7. Trembath D, Miller CR and Perry A: Gray zones in brain tumor classification: Evolving concepts. Adv Anat Pathol 15: 287-297, 2008.

8. Gay E, Lages E, Ramus C, Guttin A, El Atifi M, Dupré I, Bouamrani A, Salon C, Ratel D, Wion D, et al: The heterogeneity of meningioma revealed by multiparameter analysis: Infiltrative and non-infiltrative clinical phenotypes. Int $\mathrm{J}$ Oncol 38: 1287-1297, 2011.

9. Fritz J, Roser F, Tatagiba M and Bornemann A: The basement membrane at the tumour-brain interface of brain-invasive grade I meningiomas. Neuropathol Appl Neurobiol 31: 339-342, 2005.

10. Utsuki S, Oka H, Sato Y, Kawano N, Tsuchiya B, Kobayashi I and Fujii K: Invasive meningioma is associated with a low expression of E-cadherin and beta-catenin. Clin Neuropathol 24: $8-12,2005$.

11. Suwa T, Kawano N, Oka H, Ito H and Kameya T: Invasive meningioma: A tumour with high proliferating and 'recurrence' potential. Acta Neurochir (Wien) 136: 127-131, 1995.

12. Commins DL, Atkinson RD and Burnett ME: Review of meningioma histopathology. Neurosurg Focus 23: E3, 2007.

13. Rempel SA, Ge S and Gutiérrez JA: SPARC: A potential diagnostic marker of invasive meningiomas. Clin Cancer Res 5: 237-241, 1999.

14. Nakano T, Asano K, Miura H, Itoh S and Suzuki S: Meningiomas with brain edema: Radiological characteristics on MRI and review of the literature. Clin Imaging 26: 243-249, 2002. 